\title{
Large deviations for the boundary local time of doubly reflected Brownian motion
}

\author{
Martin Forde ${ }^{\mathrm{a}}$, Rohini Kumar ${ }^{\mathrm{b}}$, Hongzhong Zhang ${ }^{\mathrm{c}}$ \\ ${ }^{a}$ Dept. Mathematics, King's College London, London WC2R 2LS \\ ${ }^{b}$ Dept. Mathematics, Wayne State University, Detroit, MI 48202. \\ ${ }^{c}$ Dept. Statistics, Columbia University, New York, NY 10027.
}

\begin{abstract}
We compute a closed-form expression for the moment generating function $\hat{f}(x ; \lambda, \alpha)=\frac{1}{\lambda} \mathbb{E}_{x}\left(e^{\alpha L_{\tau}}\right)$, where $L_{t}$ is the local time at zero for standard Brownian motion with reflecting barriers at 0 and $b$, and $\tau \sim \operatorname{Exp}(\lambda)$ is independent of $W$. By analyzing how and where $\hat{f}(x ; \cdot, \alpha)$ blows up in $\lambda$, a large-time large deviation principle (LDP) for $L_{t} / t$ is established using a Tauberian result and the Gärtner-Ellis Theorem.
\end{abstract}

Keywords: Brownian motion, Large deviation, Local time.

\section{Introduction}

Diffusion processes with reflecting barriers have found many applications in finance, economics, biology, queueing theory, and electrical engineering. In a financial context, we recall the currency exchange rate target-zone models in KRU91] (see also [SVE91, BER92, DJ94], and BAL98|), where the exchange rate is allowed the float within two barriers; asset pricing models with price caps (see [HAN99]); interest rate models with targeting by the monetary authority (e.g. [FAR03]); short rate models with reflection at zero (e.g. [GOL97, GOR04]); and stochastic volatility models (most notably the Heston and Schöbel-Zhu models). In queueing theory, diffusions with reflecting barriers arise as heavy-traffic approximations of queueing systems and reflected Brownian motions is ubiquitous in queueing models HAR85, ABA87a, ABA87b]. More recently, reflected Ornstein-Uhlenbeck(OU) and reflected affine processes have been studied as approximations of queueing systems with reneging or balking WAR03a, WAR03b]. Applications of reflected OU processes in mathematical biology are discussed in RIC87.

Doubly reflected Brownian motion also arises naturally in the solution for the optimal trading strategy in the large-time limit for an investor who is permitted to trade a safe and a risky asset under the Black-Scholes model, subject to proportional transaction costs with exponential or power utility (see GM13] and GGMS12 respectively).

The asymptotics in this article are obtained using a Tauberian theorem. Tauberian results typically allow us to deduce the large-time or tail behavior of a quantity of interest based on the behavior of its Laplace transform (see Feller Fel71] or the excellent monograph of Bingham et al.BGT87] for details). In situations where precise tail asymptotics for a distribution function are unknown but a moment generating function is available in closedform, [BF08] establish sharp tail asymptotics on logarithmic scale as applications of the standard Kohlbecker and Karamata Tauberian theorems, combined with an Esscher change of measure; their methodology is applied to various time-changed exponential Lévy models (specifically the Variance Gamma model under a Gamma-OU clock and the Normal Inverse Gaussian model with a CIR clock) and the well known Heston stochastic volatility model.

In this article, we compute a closed-form expression for the moment generating function (mgf) $\hat{f}(x ; \lambda, \alpha)=$ $\mathbb{E}_{x}\left(e^{\alpha L_{\tau}}\right)$, where $L_{t}$ is the local time at zero for standard Brownian motion with reflecting barriers at 0 and $b$, and $\tau$ is an independent exponential random variable with parameter $\lambda$. We do this by first deriving the relevant ODE and boundary conditions for $\hat{f}(x ; \lambda, \alpha)$ using an augmented filtration and computing the optional projection, and we then solve this ODE in closed form. $\hat{f}(x ; \lambda, \alpha)$ does not appear amenable to Laplace inversion; however from an analysis of the location of the pole of $\hat{f}(x ; \cdot, \alpha)$, we can compute the re-scaled $\log \operatorname{mgf} \operatorname{limit} V(\alpha)=\lim _{t \rightarrow \infty} \frac{1}{t} \log \mathbb{E}_{x}\left(e^{\alpha L_{t}}\right)$ for $\alpha \in \mathbb{R}$ using the Tauberian result in Proposition 4.3 in Kor02] via the so-called Fejér kernel. From this we then establish a large deviation principle for $L_{t} / t$ as $t \rightarrow \infty$ using the Gärtner-Ellis Theorem from large deviations theory,

Throughout the paper, we let $\mathbb{P}_{x}(\cdot)=\mathbb{P}\left(\cdot \mid X_{0}=x\right)$ denote the law of $X$ given its initial value at time 0 for any $x \in[0, b]$, and by $\mathbb{E}_{x}(\cdot)$ the expectation under $\mathbb{P}_{x}$. Further, we let $\mathbb{E} \equiv \mathbb{E}_{0}$.

The authors would like to thank Chris Rogers for his initial suggestions on how to approach this problem and Paavo Salmimen.

Email addresses: martin.forde@kcl.ac.uk (Martin Forde), rkumar@math.wayne.edu (Rohini Kumar), hzhang@stat.columbia.edu (Hongzhong Zhang) 


\section{The modelling set up}

We begin by defining the Brownian motion $X$ with two reflecting boundaries. Let $W_{t}$ be standard Brownian motion starting at 0 . Then for any $x \in[0, b]$, there is a unique pair of non-decreasing, continuous adapted processes $(L, U)$, starting at 0 , such that

$$
X_{t}=x+W_{t}+L_{t}-U_{t} \quad \in[0, b], \quad \forall t \geq 0 .
$$

such that $L$ can only increase when $X=0$ and $U_{t}$ can only increase when $X=b$. Existence and uniqueness follow easily from the more general work of Lions\&Sznitman [LS84] the earlier work of Skorokhod [Sko62], or a bare-hands proof can be given by successive applications of the standard one-sided reflection mapping using a sequence of stopping times (see [Wil92].)

It can be shown that

$$
\begin{gathered}
\lim _{t \rightarrow \infty} L_{t} / t=\mathbb{E}\left(L_{\tau^{b}+\tau^{\prime}}\right) / \mathbb{E}\left(\tau^{b}+\tau^{\prime}\right), \quad \lim _{t \rightarrow \infty} U_{t} / t=\mathbb{E}\left(U_{\tau^{b}+\tau^{\prime}}\right) / \mathbb{E}\left(\tau^{b}+\tau^{\prime}\right), \\
\lim _{t \rightarrow \infty} \frac{1}{t} \operatorname{Var}\left(L_{t}\right)=\sigma_{L}^{2}, \quad \lim _{t \rightarrow \infty} \frac{1}{t} \operatorname{Var}\left(U_{t}\right)=\sigma_{U}^{2},
\end{gathered}
$$

where $\tau^{b}=\inf \left\{t: X_{t}=b\right\}, \tau^{\prime}=\inf \left\{t \geq \tau^{b}: X_{t}=0\right\}$ (see Wil92]) for some non-negative constants $\sigma_{L}, \sigma_{U}$.

Proposition 2.1. Let $\tau$ denote an independent exponential random variable with parameter $\lambda$. Then for $\alpha<0$,

$$
\hat{f}(x) \equiv \hat{f}(x ; \lambda, \alpha):=\frac{1}{\lambda} \mathbb{E}_{x}\left(e^{\alpha L_{\tau}}\right)=\frac{1}{\lambda} \int_{0}^{\infty} e^{-\lambda t} \mathbb{E}_{x}\left(e^{\alpha L_{t}}\right) d t
$$

is smooth on $(0, b)$ and satisfies the following $O D E$

$$
\frac{1}{2} \hat{f}_{x x}=\lambda \hat{f}-1, \hat{f}_{x}(0)+\alpha \hat{f}(0)=\hat{f}_{x}(b)=0 .
$$

Proof. We first show that $\hat{f} \in C^{\infty}(0, b)$. To this end, note that for $x \in[0, b]$,

$$
\mathbb{E}_{x}\left(e^{\alpha L_{\tau}}\right)=\mathbb{P}_{x}\left(\tau>H_{0}\right) \mathbb{E}_{0}\left(e^{\alpha L_{\tau}}\right)+\mathbb{P}_{x}\left(\tau \leq H_{0}\right)
$$

where $H_{x}=\inf \left\{t: X_{t}=x\right\}$ is the first hitting time to $x$. The law of $\left(b-X_{t} ; t \in\left[0, H_{0}\right]\right)$ given $X_{t}=x$ is the same as that of $\left(\left|W_{t}\right| ; t \in\left[0, H_{b}\right]\right)$ given $\left|W_{0}\right|=b-x$. Thus by Eq. 2.0.1 on page 355 of [BS02] we have

$$
\mathbb{P}_{x}\left(\tau>H_{0}\right)=\mathbb{E}_{x}\left(e^{-\lambda H_{0}}\right)=\frac{\cosh ((b-x) \sqrt{2 \lambda})}{\cosh (b \sqrt{2 \lambda})} .
$$

It follows that

$$
\mathbb{E}_{x}\left(e^{\alpha L_{\tau}}\right)=\frac{\cosh ((b-x) \sqrt{2 \lambda})}{\cosh (b \sqrt{2 \lambda})}\left[\mathbb{E}_{0}\left(e^{\alpha L_{\tau}}\right)-1\right]+1
$$

That is,

$$
\hat{f}(x)=\frac{\cosh ((b-x) \sqrt{2 \lambda})}{\cosh (b \sqrt{2 \lambda})}\left(\hat{f}(0)-\frac{1}{\lambda}\right)+\frac{1}{\lambda}, \forall x \in[0, b] .
$$

It can then be easily seen from (2) that $\hat{f} \in C^{\infty}(0, b)$.

To show that $\hat{f}$ satisfies (1) and the boundary conditions, we construct a martingale that is adapted to the filtration generated by $X$. More specifically, we introduce the natural filtration $\mathcal{F}_{t}=\sigma\left(X_{s} ; s \leq t\right)$ and the augmented filtration $\overline{\mathcal{F}}_{t}=\mathcal{F}_{t} \vee \sigma\left(\mathbf{1}_{\{\tau<t\}}\right)$, where $\sigma\left(\mathbf{1}_{\{\tau<t\}}\right)$ is the sigma algebra generated by $\mathbf{1}_{\{\tau<t\}}$. Then we have a uniformly bounded, and hence uniformly integrable $\overline{\mathcal{F}}_{t}$-martingale:

$$
\bar{M}_{t}:=\mathbb{E}\left(e^{\alpha L_{\tau}} \mid \overline{\mathcal{F}}_{t}\right)=\mathbf{1}_{\{\tau<t\}} e^{\alpha L_{\tau}}+\mathbf{1}_{\{\tau \geq t\}} e^{\alpha L_{t}} \mathbb{E}_{X_{t}}\left(e^{\alpha L_{\tau}}\right)=\mathbf{1}_{\{\tau<t\}} e^{\alpha L_{\tau}}+\mathbf{1}_{\{\tau \geq t\}} e^{\alpha L_{t}} \lambda \hat{f}\left(X_{t}\right) .
$$

We now define the optional projection of $\bar{M}_{t}$ : using the fact that $X$ and $\tau$ are independent, we have

$$
M_{t}=\mathbb{E}\left(\bar{M}_{t} \mid \mathcal{F}_{t}\right)=\lambda \int_{0}^{t} e^{\alpha L_{s}-\lambda s} d s+e^{\alpha L_{t}-\lambda t} \lambda \hat{f}\left(X_{t}\right)
$$


Further, $M_{t}$ is a $\mathcal{F}_{t}$-martingale, in that for all $t>s$ we have

$$
\mathbb{E}\left(M_{t} \mid \mathcal{F}_{s}\right)=\mathbb{E}\left(\mathbb{E}\left(\overline{M_{t}} \mid \mathcal{F}_{t}\right) \mid \mathcal{F}_{s}\right)=\mathbb{E}\left(\bar{M}_{t} \mid \mathcal{F}_{s}\right)=\mathbb{E}\left(\mathbb{E}\left(\bar{M}_{t} \mid \overline{\mathcal{F}}_{s}\right) \mid \mathcal{F}_{s}\right)=\mathbb{E}\left(\bar{M}_{s} \mid \mathcal{F}_{s}\right)=M_{s}
$$

Applying Itō's lemma to $M_{t}$, we have that

$$
d M_{t}=e^{\alpha L_{t}-\lambda t}\left[\lambda d t+\lambda \hat{f}\left(X_{t}\right)\left(\alpha d L_{t}-\lambda d t\right)+\frac{1}{2} \lambda \hat{f}_{x x}\left(X_{t}\right) d t+\lambda \hat{f}_{x}\left(X_{t}\right)\left(d W_{t}+d L_{t}-d U_{t}\right)\right] .
$$

But for $M_{t}$ to be a martingale, we must have

$$
\frac{1}{2} \hat{f}_{x x}(x)-\lambda \hat{f}(x)+1=0, \quad \hat{f}_{x}(0)+\alpha \hat{f}(0)=0, \hat{f}_{x}(b)=0 .
$$

This completes the proof.

Solving the ODE in Proposition 2.1 we obtain the following result:

\section{Proposition 2.2.}

$$
\hat{f}(x ; \lambda, \alpha)=\frac{1}{\lambda}+e^{x \sqrt{2 \lambda}} A_{\lambda}(\alpha)+e^{-x \sqrt{2 \lambda}} B_{\lambda}(\alpha)
$$

for $\lambda>0, \alpha<0$, where

$$
A_{\lambda}(\alpha)=\frac{\alpha e^{-b \sqrt{2 \lambda}} / \cosh (b \sqrt{2 \lambda})}{2 \lambda\left[\alpha^{*}(\lambda)-\alpha\right]}, B_{\lambda}(\alpha)=e^{2 \sqrt{2 \lambda} b} A_{\lambda}(\alpha), \alpha^{*}(\lambda)=\sqrt{2 \lambda} \tanh (b \sqrt{2 \lambda}) .
$$

Remark 2.3. Observe that the expression for $\hat{f}(x)$ involves $\sqrt{\lambda}$, which has a branch point at $\lambda=0$. However, $\hat{f}$ remains a continuous function across the branch cut at $\lambda=0$; thus $\hat{f}$ is an analytic function of $\lambda$ in some punctured disc about $\lambda=0$. As $\lim _{\lambda \rightarrow 0} \lambda \cdot \hat{f}(\lambda)=0$, we conclude that $\lambda=0$ is a removable singularity.

Remark 2.4. It can be verified that $\alpha^{*}(\cdot)$ in (4) is a strictly increasing mapping from $[0, \infty)$ onto $[0, \infty)$. Further, we may analytically extend $\alpha^{*}(\cdot)$ to get a strictly increasing, strictly concave, smooth real-valued function that maps $\left(-\frac{\pi^{2}}{8 b^{2}}, \infty\right)$ onto $\mathbb{R}$.

\section{Large-time asymptotics}

In this section, we characterize the large-time behaviour of $L_{t}$. To this end, let us consider the inverse of $\alpha^{*}$, $V(\alpha):=\left(\alpha^{*}\right)^{-1}(\alpha)$ for $\alpha \in \mathbb{R}$. From Remark 2.4 we know that $V$ is a strictly increasing, strictly convex smooth function, with range $\left(-\frac{\pi^{2}}{8 b^{2}}, \infty\right)$.

Lemma 3.1. The equality (3) also holds for all $\alpha \in \mathbb{R}, \lambda \in \mathbb{C}$ such that $\Re(\lambda)>V(\alpha)$.

Proof. See Appendix A

Proposition 3.2. We have the following large-time behaviour for the moment generating function of $L_{t}$ :

$$
\lim _{t \rightarrow \infty} \frac{1}{t} \log \mathbb{E}_{x}\left(e^{\alpha L_{t}}\right)=V(\alpha)<\infty \forall \alpha \in \mathbb{R} .
$$

Proof. See Appendix B

Remark 3.3. Note that $V(\cdot)$ does not depend on the starting value $x$, due to the ergodicity of $X$.

The following lemma will be needed in the statement of the large deviation principle in the theorem that follows.

Lemma 3.4. (a) Define $V^{*}(x):=\sup _{\alpha \in \mathbb{R}}[\alpha x-V(\alpha)]$ for all $x \geq 0$. Then we have

$$
V^{*}(x)=\left\{\begin{array}{ll}
x \alpha^{*}\left(\lambda^{*}\right)-\lambda^{*}, & \text { for } x>0 \\
\pi^{2} /\left(8 b^{2}\right), & \text { for } x=0
\end{array},\right.
$$

where $\lambda^{*}=\lambda^{*}(x)$ is the unique solution of $\left(\alpha^{*}\right)^{\prime}(\lambda)=1 / x$ for fixed $x>0$.

(b) $V^{*} \in C([0, \infty)) \cap C^{1}((0, \infty))$ and $V^{*}$ is a strictly convex function on $(0, \infty)$.

(c) $V^{*}$ attains its minimum value of zero uniquely at $x^{*}=\frac{1}{2 b}$. 
Proof. See Appendix C

Theorem 3.5. $L_{t} / t$ satisfies a large deviation principle on $[0, \infty)$ as $t \rightarrow \infty$ with a strictly convex rate function $V^{*}(x)$.

Proof. From Lemma 3.4 we know that $V^{*}$ is a strictly convex function on $(0, \infty)$. Hence the set of exposed points of $V^{*}$ is $(0, \infty)$ (see Definition 2.3.3 in [DZ98]), and since $D_{V}^{0}=(-\infty, \infty)$, the exposing hyperplane will always lie in $D_{V}^{0}$. Therefore, by the Gärtner-Ellis Theorem (see Theorem 2.3.6 in [DZ98]), $L_{t} / t$ satisfies the LDP with convex rate function $V^{*}(x)$.

\section{Appendix A. Proof of Lemma 3.1}

Recall from Propositions 2.1 and 2.2 that, for $\alpha<0$ and $\lambda>0$,

$$
\int_{0}^{\infty} e^{-\lambda t} \mathbb{E}_{x}\left(e^{\alpha L_{t}}\right) d t=\hat{f}(x ; \lambda, \alpha)=\frac{1}{\lambda}+e^{x \sqrt{2 \lambda}} A_{\lambda}(\alpha)+e^{-x \sqrt{2 \lambda}} B_{\lambda}(\alpha)
$$

where $A_{\lambda}(\alpha)=\frac{\alpha e^{-b \sqrt{2 \lambda}} / \cosh (b \sqrt{2 \lambda})}{2 \lambda\left[\alpha^{*}(\lambda)-\alpha\right]}$ and $B_{\lambda}(\alpha)=e^{2 \sqrt{2 \lambda} b} A_{\lambda}(\alpha)$. We wish to show that (B-1) still holds for a wider range of $\alpha$ and $\lambda$ values using analytic continuation. We first note that $\hat{f}$ has a singularity when $\alpha=\alpha^{*}(\lambda)$, and by Theorems $5 \mathrm{a}$ and $5 \mathrm{~b}$ on page 57 in Wid46], we know that the abscissa of convergence for a Laplace transform is a point of singularity and the Laplace transform is analytic in its region of convergence.

We are interested in the values of $\alpha \in \mathbb{R}$ and $\lambda \in \mathbb{C}$ such that the Laplace transform $\hat{f}(x ; \lambda, \alpha)=\int_{0}^{\infty} e^{-\lambda t} \mathbb{E}_{x}\left(e^{\alpha L_{t}}\right) d t$ is finite. We recall the following fact: for any fixed $x \in[0, b]$,

(†) $\hat{f}(x ; \lambda, \alpha)<\infty$ for $\alpha<0$ and $\lambda>0$.

We now proceed in three stages:

- Fix $\lambda>0$ (so $\lambda \in \mathbb{R}$ ). We apply the Widder results with $\alpha$ as the Laplace variable, i.e. we consider

$$
\mathbb{E}\left(e^{\alpha L_{\tau}}\right)=\int_{0}^{\infty} e^{\alpha y} d F(y)
$$

where $F(y)$ is the distribution function of $L_{\tau}$. By $(\dagger)$, the region of convergence is non-empty. We can then extend the region of convergence up to $\alpha^{*}(\lambda)>0$, as $\alpha^{*}(\lambda)$ is the point of singularity.

- Fix $\alpha<0$. We apply the Widder results again, but we now take $\lambda$ as the Laplace variable. By $(\dagger)$, the region of convergence is non-empty. According to Widder, the abscissa of convergence (say $\lambda_{c}$ ) is a point of singularity and $\hat{f}(x ; \lambda, \alpha)$ is analytic in $\lambda$ when $\Re(\lambda)>\lambda_{c}$. So we are looking at a point of singularity on the real line, and this is the value of $\lambda_{c}$ that satisfies $\alpha^{*}\left(\lambda_{c}\right)=\alpha$. Or, in other words, $\lambda_{c}=\left(\alpha^{*}\right)^{-1}(\alpha)=V(\alpha)$. Thus, by Widder, $\hat{f}(x ; \lambda, \alpha)$ is finite when $\Re(\lambda)>V(\alpha)$.

- Fix $\alpha>0$. We apply Widder's theorem using $\lambda$ as the Laplace variable. By the first bullet point, we know that there exists some $\lambda \in \mathbb{R}$ (such that $\alpha<\alpha^{*}(\lambda)$ ), for which $\hat{f}(x ; \lambda, \alpha)$ is finite. Hence, the region of convergence of $\hat{f}(x ; \lambda, \alpha)$ is non-empty for this $\alpha$. Then, by Widder, the abscissa of convergence $\lambda_{c}$ is a point of singularity and $\hat{f}(x ; \lambda, \alpha)$ is analytic for $\Re(\lambda)>\lambda_{c}$. The singularity is at $\alpha=\alpha^{*}(\lambda)$. Solving for points of singularity on the real line i.e. solving for $\lambda_{c}$ in $\alpha=\alpha^{*}\left(\lambda_{c}\right)$, gives us $\lambda_{c}=V(\alpha)$ and so $\hat{f}(x ; \lambda, \alpha)$ converges when $\Re(\lambda)>\lambda_{c}=V(\alpha)$.

This gives the region of $\lambda$ and $\alpha$ for which $\hat{f}(x ; \lambda, \alpha)$ converges: for every $\alpha \in \mathbb{R}$ and $\lambda \in \mathbb{C}$ such that $\Re(\lambda)>V(\alpha)$, and $\hat{f}(x ; \lambda, \alpha)$ is analytic in this region.

\section{Appendix B. Proof of Proposition 3.2}

From the known large-time behaviour of the local time of standard Brownian motion, we expect that $\mathbb{E}_{x}\left(e^{\alpha L_{t}}\right) \sim$ const. $\times e^{U(\alpha) t}$ as $t \rightarrow \infty$, for some non-decreasing function $U(\alpha)$ to be determined. Then as $t \rightarrow \infty$,

$$
\hat{f}(x ; \lambda, \alpha)=\int_{0}^{\infty} e^{-\lambda t} \mathbb{E}_{x}\left(e^{\alpha L_{t}}\right) d t \sim \int_{0}^{\infty} e^{-\lambda t} \text { const. } \times e^{U(\alpha) t} d t,
$$


and $\hat{f}(x ; \lambda, \alpha)$ blows up when $\lambda=U(\alpha)$ (for $\alpha$ fixed). But we know that $\hat{f}(x ; \lambda, \alpha)$ blows up at $\alpha=\alpha^{*}(\lambda)$; thus we expect that $\lambda=U\left(\alpha^{*}(\lambda)\right)$, i.e. $U(\alpha)=\left(\alpha^{*}\right)^{-1}(\alpha)=V(\alpha)$. We now make this statement rigorous using a variant of Ikehara's Tauberian Theorem (see e.g. Theorem 17 on page 233 in Widder Wid46]).

We first define a positive function $v$ on $\mathbb{R}$ :

$$
v(t) \equiv v(t ; x, \alpha):=\mathbf{1}_{t \geq 0} e^{-V(\alpha) t} \mathbb{E}_{x}\left(e^{\alpha L_{t}}\right) .
$$

Then the Laplace transform of $v$ is given by

$$
\hat{v}(\lambda)=\int_{0}^{\infty} e^{-\lambda t} v(t) d t=\int_{0}^{\infty} e^{-(\lambda+V(\alpha)) t} \mathbb{E}_{x}\left(e^{\alpha L_{t}}\right) d t=\hat{f}_{\lambda+V(\alpha), \alpha}(x),
$$

which, by Lemma 3.1 is analytic for all $\lambda \in \mathbb{C}$ such that $\Re(\lambda)>0$. We now need to characterize how $\hat{v}(\lambda)$ blows up as $\Re(\lambda) \downarrow 0$. To this end, looking at the expression for $A_{\lambda}(\alpha)$, we notice that $A_{\lambda}(\alpha)$ has a pole at $\lambda=V(\alpha) \in\left(-\frac{\pi^{2}}{8 b^{2}}, \infty\right)$, and is analytic elsewhere for $\Re(\lambda)>-\frac{\pi^{2}}{8 b^{2}}$ (see Remark 2.3). It is also easily seen that, $\alpha^{* \prime}(\lambda)>0$ for all $\lambda \in\left(-\frac{\pi^{2}}{8 b^{2}}, \infty\right)$. Hence, by the Laurent expansion of $\hat{v}(\lambda)$ at 0 , there exists a function $g(\lambda)$, which is analytic for all $\lambda \in \mathbb{C}$ with $\Re(\lambda)>-\varepsilon$ and $|\Im(\lambda)| \leq c$ for some constants $\varepsilon, c>0$, such that

$$
\hat{v}(\lambda)=\frac{C}{\lambda}+g(\lambda)
$$

for some constant $C$ which we find to be positive $(C$ is the residue of $\hat{v}$ at $\lambda=0) . g(x+i y)$ is continuous on $\mathcal{D}:=\{(x, y):|x| \leq \varepsilon,|y| \leq c\}$, thus $g(x+i y)$ is uniformly continuous on $\mathcal{D}$, so $g(x+i y) \rightarrow g(i y)$ uniformly as $x \downarrow 0$ for any fixed $y \in[-c, c]$. Moreover, for any $x>0$

$$
\int_{-c}^{c}\left|\hat{v}(x+i y)-\frac{C}{x+i y}-g(i y)\right| d y=\int_{-c}^{c}|g(x+i y)-g(i y)| d y
$$

Since $g$ is analytic everywhere and uniformly continuous, if we take the limit as $x \rightarrow 0$, the above integral converges to 0 , so the function $g(x+i \cdot)$ also converge to $g(i \cdot)$ in $\mathbb{L}^{1}([-c, c])$, as $x \downarrow 0$.

We can now apply Proposition 4.3 in [Kor02] to obtain that for the "Fejér kernel" $K(t)=\frac{1-\cos t}{\pi t^{2}}$,

$$
\lim _{t \rightarrow \infty} \int_{-\infty}^{c t} v\left(t-\frac{s}{c}\right) \cdot K(s) d s=C
$$

We now proceed as in the proof of Theorem 4.2 in $\underline{\text { Kor02 }}$ to show that $v(t)=O(1)$ as $t \rightarrow \infty$.

1. $\alpha>0$. In this case we know that $\mathbb{E}_{x}\left(e^{\alpha L_{t}}\right)$ is non-decreasing, so $v(t) \geq v(s) e^{V(\alpha)(s-t)}$ for all $t \geq s \geq 0$. For any fixed $a>0$, using (C-2) we have that

$$
C=\lim _{t \rightarrow \infty} \int_{-\infty}^{c t} v\left(t-\frac{s}{c}\right) \cdot K(s) d s \geq \limsup _{t \rightarrow \infty} \int_{-a}^{a} v\left(t-\frac{s}{c}\right) \cdot K(s) d s \geq \limsup _{t \rightarrow \infty} v\left(t-\frac{a}{c}\right) e^{-2 V(\alpha) \frac{a}{c}} \int_{-a}^{a} K(s) d s,
$$

which implies that

$$
\limsup _{t \rightarrow \infty} v(t) \leq \frac{e^{2 V(\alpha) \frac{a}{c}}}{\int_{-a}^{a} K(s) d s} C<\infty .
$$

Hence, there exists a constant $M>0$ such that $v(t) \leq M$ for all $t$. Similarly, for any fixed $a>0$, we have

$$
\begin{aligned}
\liminf _{t \rightarrow \infty} v\left(t+\frac{a}{c}\right) e^{2 V(\alpha) \frac{a}{c}} \int_{-a}^{a} K(s) d s \geq \liminf _{t \rightarrow \infty} \int_{-a}^{a} v\left(t-\frac{s}{c}\right) K(s) d s \\
=\liminf _{t \rightarrow \infty}\left(\int_{0}^{c t}+\int_{c t}^{\infty}-\int_{-\infty}^{-a}-\int_{a}^{\infty}\right) v\left(t-\frac{s}{c}\right) K(s) d s \geq \liminf _{t \rightarrow \infty}\left(\int_{0}^{c t}+\int_{c t}^{\infty}\right) v\left(t-\frac{s}{c}\right) K(s) \\
\quad-\limsup _{t \rightarrow \infty} \int_{-\infty}^{-a} v\left(t-\frac{s}{c}\right) K(s)-\limsup _{t \rightarrow \infty} \int_{a}^{\infty} v\left(t-\frac{s}{c}\right) K(s) \geq C-\frac{4 M}{\pi} \int_{a}^{\infty} \frac{1}{s^{2}} d s=C-\frac{4 M}{\pi a},
\end{aligned}
$$

where we have used (C-2) and the fact that $0 \leq K(t) \leq \frac{2}{\pi t^{2}}$ in the last inequality. Hence, for $a>0$ sufficiently large, we have

$$
\liminf _{t \rightarrow \infty} v(t) \geq \frac{e^{-2 V(\alpha) \frac{a}{c}}}{\int_{-a}^{a} K(s) d s}(C-4 M / \pi a)>0 .
$$


2. $\alpha<0$. In this case we know that $\mathbb{E}_{x}\left(e^{\alpha L_{t}}\right)$ is non-increasing, so $v(t) \leq v(s) e^{V(\alpha)(s-t)}$ for all $t \geq s \geq 0$. Using the same argument as above, we have, for any fixed $a>0$,

$$
\begin{aligned}
C e^{2 V(\alpha) \frac{a}{c}} & \geq \limsup _{t \rightarrow \infty} v\left(t+\frac{a}{c}\right) \int_{-a}^{a} K(s) d s, \\
\left(C-\frac{4 M}{\pi a}\right) e^{-2 V(\alpha) \frac{a}{c}} & \leq \liminf _{t \rightarrow \infty} v\left(t-\frac{a}{c}\right) \int_{-a}^{a} K(s) d s .
\end{aligned}
$$

Hence for $a>0$ sufficiently large, we have

$$
0 \leq \frac{e^{-2 V(\alpha) \frac{a}{c}}}{\int_{-a}^{a} K(s) d s}(C-4 M / \pi a) \leq \liminf _{t \rightarrow \infty} v(t) \leq \limsup _{t \rightarrow \infty} v(t) \leq \frac{e^{2 V(\alpha) \frac{a}{c}}}{\int_{-a}^{a} K(s) d s} C<\infty .
$$

Hence, by Proposition 4.3 in [Kor02], the result follows.

\section{Appendix C. Proof of Lemma 3.4}

We break the proof into three parts:

(a) Computing the Legendre transform of $V$ boils down to solving $V^{\prime}(\alpha)=x$. But this is the same as solving $\left(V^{-1}\right)^{\prime}(\lambda)=\frac{1}{x}$ for $\lambda$, when $x>0$. Recall that $V^{-1}(\cdot)=\alpha^{*}(\cdot)$ is known in closed form. Since $\left(\alpha^{*}\right)^{\prime \prime}(\lambda)<0$ for all $\lambda$ (from Remark 2.4), by the Inverse function theorem, $\lambda^{*}(x):=\left(\left(\alpha^{*}\right)^{\prime}\right)^{-1}(1 / x)$ is well-defined and $\lambda^{*} \in C^{1}((0, \infty))$. Using the fact that $\alpha^{*}\left(\lambda^{*}\right)=V^{-1}\left(\lambda^{*}\right)$, we have

$$
V^{*}(x)=x \alpha^{*}-V\left(\alpha^{*}\right)=x \alpha^{*}\left(\lambda^{*}(x)\right)-\lambda^{*}(x) .
$$

When $x=0$, the definition of $V^{*}$ in Lemma 3.4 gives us $V^{*}(0)=\sup _{\alpha \in \mathbb{R}}\{-V(\alpha)\}=-\inf _{\alpha \in \mathbb{R}}\{V(\alpha)\}=$ $-\lim _{\alpha \rightarrow-\infty} V(\alpha)=\pi^{2} /\left(8 b^{2}\right)$, where the last two equalities hold because $V$ is a monotonically increasing function with range $\left(-\pi^{2} /\left(8 b^{2}\right), \infty\right)$.

(b) By the Inverse function theorem, we know that $\lambda^{*} \in C^{1}((0, \infty))$ and so is $\alpha^{*}$, thus $V^{*} \in C^{1}((0, \infty))$. It is easy to check that $\lim _{x \downarrow 0}\left\{x \alpha^{*}\left(\lambda^{*}(x)\right)-\lambda^{*}(x)\right\}=\pi^{2} /\left(8 b^{2}\right)=V^{*}(0)$, which gives continuity of $V^{*}$ up to the boundary $x=0$. Using (5), we obtain

$$
\begin{aligned}
\left(V^{*}\right)^{\prime}(x) & =\alpha^{*}\left(\lambda^{*}(x)\right)+x \cdot\left(\alpha^{*}\right)^{\prime}\left(\lambda^{*}(x)\right) \cdot\left(\lambda^{*}\right)^{\prime}(x)-\left(\lambda^{*}\right)^{\prime}(x) \\
& =\alpha^{*}\left(\lambda^{*}(x)\right)+x \cdot \frac{1}{x} \cdot\left(\lambda^{*}\right)^{\prime}(x)-\left(\lambda^{*}\right)^{\prime}(x)=\alpha^{*}\left(\lambda^{*}(x)\right)
\end{aligned}
$$

$\left(V^{*}\right)^{\prime}(x)=\alpha^{*}\left(\lambda^{*}(x)\right)$. Thus we have (using again $\left.\left(\alpha^{*}\right)^{\prime \prime}<0\right)$

$$
\left(V^{*}\right)^{\prime \prime}(x)=\left(\alpha^{*}\right)^{\prime}\left(\lambda^{*}(x)\right) \cdot\left(\lambda^{*}\right)^{\prime}(x)=\frac{1}{x} \cdot\left(\left(\alpha^{*}\right)^{\prime-1}\right)^{\prime}\left(\frac{1}{x}\right) \cdot-\frac{1}{x^{2}}=-\frac{1}{x^{3}} \cdot \frac{1}{\left(\alpha^{*}\right)^{\prime \prime}\left(\lambda^{*}(x)\right)}>0 .
$$

(c) Since $V^{*}$ is strictly convex, it has a unique minimum. The unique minimum of $V^{*}$ occurs at $x^{*}=\left(\left(V^{*}\right)^{\prime}\right)^{-1}(0)=$ $V^{\prime}(0)=1 / \alpha^{* \prime}(0)=\frac{1}{2 b}$

\section{References}

[ABA87a] Abate, J., Whitt, W., "Transient behavior of regulated brownian motion, I: starting at the origin", Advances in Applied Probability, 19, 560-598, 1987.

[ABA87b] Abate, J., Whitt, W., "Transient behavior of regulated brownian motion, II: non-zero initial conditions", Advances in Applied Probability, 19, 599-631, 1987.

[BAL98] Ball, C., Roma, A., "Detecting mean reversion within reflecting barriers: application to the european exchange rate mechanism.", Applied Mathematical Finance, 5(1), 1-15, 1998.

[BW92] Berger, W. and Whitt W., "The Brownian approximation of rate-control throttles and the G/G/1/C queue", Dynamic Discrete Event Systems:Theory and Applications, 2, 7-60, 1992.

[BER92] Bertola, G., Caballero, R., "Target zones and realignments", The American Economic Review, 520-536, 1992. 
[BF08] Benaim, S. and P.Friz, "Smile Asymptotics II: Models with Known MGF", J. Appl. Probab., Volume 45, Number 1 (2008), 16-32.

[BGT87] Bingham, N.H., Goldie, C.M., Teugels, J.L., "Regular Variation", CUP 1987.

[BS02] Borodin, A.N. and P.Salminen, "Handbook of Brownian Motion - Facts and Formulae", Birkhauser, 2002.

[DJ94] De Jong, F., "A univariate analysis of EMS exchange rates using a target zone model", Journal of Applied Econometrics, 9(1),31-45, 1994.

[DZ98] Dembo, A. and O.Zeitouni, "Large deviations techniques and applications", Jones and Bartlet publishers, Boston, 1998.

[FAR03] Farnsworth, H. and Bass, R., "The term structure with semi-credible targeting.", The Journal of Finance, $58(2), 839-866,2003$.

[Fel71] Feller, W., "An Introduction to Probability Theory and Its Applications: Vol. 2", John Wiley and Sons, New York, 1971.

[GGMS12] Gerhold, S., P.Guasoni, J.Muhle-Karbe, W.Schachermayer, "Transaction costs, trading volume, and the liquidity premium", to appear in Finance and Stochastics.

[GOL97] Goldstein R., Keirstead, W., "On the term structure of interest rates in the presence of reflecting and absorbing boundaries.", Fisher College of Business, The Ohio State University, 1-36, 1997.

[GOR04] Gorovoi, V. and Linetsky, V., "Black's model of interest rates as options, eigenfunction expansions and Japanese interest rates" Mathematical finance, 14(1),49-78, 2004.

[GM13] Guasoni, P. and J.Muhle-Karbe, "Long Horizons, High Risk Aversion, and Endogeneous Spreads", to appear in Mathematical Finance.

[HAN99] Hanson, S.D., Myers, R.J. and Hilker, J.H. "Hedging with futures and options under a truncated cash price distribution." Journal of Agricultural and Applied Economics, 31(3),449-460, 1999.

[HAR85] Harrison, M. "Brownian motion and stochastic flow systems." Wiley New York, 1985.

[KRU91] Krugman, P.R. "Target zones and exchange rate dynamics." The Quarterly Journal of Economics, 106(3), 669-682, 1991.

[Kor02] Korevaar, J. "A century of complex Tauberian theory", Bulletin of the American Mathematical Society, Vol. 39, 2002, pp. 475-531.

[LS84] Lions, P.L. and A.S.Sznitman, "Stochastic differential equations with reflecting boundary conditions", Comm. Pure Appl. Math. 37, 511-537, 1984.

[RIC87] Ricciardi, L.M. and Sacerdote, L., "On the probability densities of an Ornstein-Uhlenbeck process with a reflecting boundary.", Journal of Applied Probability, 355-369, 1987.

[Sko62] A.V.Skorohod. Stochastic equations for diffusion processes with boundaries. II. Teor. Verojatnost. i Primenen., 7:5, 25, 1962.

[SVE91] Svensson, L.E.O., "The term structure of interest rate differentials in a target zone. Theory and Swedish data." Journal Monetary Economics, 28,87-116, 1991.

[WAR03a] Ward, A. and Glynn, P.W., "A diffusion approximation for a Markovian queue with reneging." Queueing Systems, 43(1-2), 103-128, 2003.

[WAR03b] Ward, A. and Glynn, P.W., "Properties of the reflected Ornstein-Uhlenbeck process." Queueing Systems, $44(2), 109-123,2003$.

[Wid46] Widder, D.V., "The Laplace Transform", Dover Publication, 1946.

[Wil92] Williams, R.J., "Asymptotic Variance Parameters for the Boundary Local Times of Reflected Brownian Motion on a Compact Interval", Journal of Applied Probability, 29(4), 996-1002, 1992. 